\title{
On generalized metric properties of the Fell hyperspace
}

Article in Annali di Matematica Pura ed Applicata · January 2014

DOI: $10.1007 /$ s10231-014-0418-2

CITATION

1

2 authors:

\section{L'ubica Holá}

Slovak Academy of Sciences

108 PUBLICATIONS 513 CITATIONS

SEE PROFILE
READS

37

\section{Laszlo Zsilinszky}

University of North Carolina at Pembroke 42 PUBLICATIONS 152 CITATIONS

SEE PROFILE

Some of the authors of this publication are also working on these related projects: 


\title{
On generalized metric properties of the Fell hyperspace
}

\author{
L’ubica Holá • László Zsilinszky
}

Received: 8 January 2014 / Accepted: 2 April 2014

(C) Fondazione Annali di Matematica Pura ed Applicata and Springer-Verlag Berlin Heidelberg 2014

\begin{abstract}
It is shown that if $X$ contains a closed uncountable discrete subspace, then the Tychonoff plank embeds in the hyperspace $C L(X)$ of the non-empty closed subsets of $X$ with the Fell topology $\tau_{F}$ as a closed subspace. As a consequence, a plethora of properties is proved to be equivalent to normality and metrizability, respectively, of $\left(C L(X), \tau_{F}\right)$. Countable paracompactness, pseudonormality and other weak normality properties of the Fell topology are also characterized.
\end{abstract}

Keywords Fell topology · Vietoris topology - Tychonoff plank · Extent - Spread . Metrizable $\cdot$ Lindelöf · (hereditary) normal · Monotonically normal $\cdot \delta$-Normal . Pseudonormal $\cdot$ Countably paracompact

Mathematics Subject Classification (2010) $\quad 54 \mathrm{~B} 20 \cdot 54 \mathrm{C} 25 \cdot 54 \mathrm{D} 15 \cdot 54 \mathrm{D} 20$

\section{Introduction}

The Fell topology $\tau_{F}$ on the space of (non-empty) closed subsets of a topological space $X$ is a fundamental construct due to its usefulness in various areas of mathematics and applications $[3,4,19]$. Since the Fell topology is a hit-and-miss type topology (i.e., a typical base element for $\tau_{F}$ consists of closed sets that hit finitely many open subsets of $X$ and miss a compact subset of $X$ ), it has frequently been compared to another classical well-studied hit-and-miss

L'ubica Holá would like to thank the support of the grant APVV-0269-11 and Vega 2/0018/13.

L. Holá

Academy of Sciences, Institute of Mathematics, Štefánikova 49, 81473 Bratislava, Slovakia

e-mail: hola@mat.savba.sk

L. Zsilinszky $(\varangle)$

Department of Mathematics and Computer Science, The University of North Carolina at Pembroke, Pembroke, NC28372, USA

e-mail: laszlo@uncp.edu 
topology, the Vietoris topology $\tau_{V}$, for motivation to obtain results and ideas about $\tau_{F}$. This is far from an automatic transfer of properties and results (see e.g., characterizations of normality of these topologies [14,17,27]); on the other side, for various properties, one can find common characterizations of even general hit-and-miss topologies (e.g., for separation, countability axioms, metrizability, completeness [3,28]).

In this paper, we continue studying the Fell topology and obtain new results about various generalized metric properties of $\tau_{F}$. The motivation came from analogous properties of the Vietoris topology (results of several authors spread in the literature); however, instead of separately proving these results for the Fell topology, we present a technique that produces the proofs simultaneously. The technique relies on some embedding results presented in Sect. 2, which allow us, in Sect. 3, to prove the equivalence of a large number of properties of $\tau_{F}$ to its normality, and we also look at properties that are strictly weaker than normality, but we show they still coincide with normality for $\tau_{F}$, fixing a result of [20] in the process. Finally, in Sect. 4, we characterize more generalized metric properties of $\tau_{F}$ showing them equivalent to its metrizability through a method that ties the analogous (known) results of the Vietoris topology to those of the Fell topology.

First, we introduce some notation and terminology: throughout the paper, $X$ is a Hausdorff space. Denote by $2^{X}$ (resp. $\left.C L(X)\right)$ the (non-empty) closed subsets of $X$. For $S \subseteq X$, put

$$
S^{-}=\left\{A \in 2^{X}: A \cap S \neq \emptyset\right\}, S^{+}=\left\{A \in 2^{X}: A \subseteq S\right\} .
$$

For a finite collection $\mathscr{S}$ of subsets of $X$ denote

$$
\mathscr{S}^{-}=\bigcap_{S \in \mathscr{S}} S^{-}
$$

The Vietoris topology [22] $\tau_{V}$ on $2^{X}$ has as a subbase elements of the form $U^{-}, U^{+}$, where $U$ is open in $X$. The Fell topology [11] $\tau_{F}$ on $2^{X}$ has as a subbase the collection

$$
\left\{U^{-}: U \text { open in } X\right\} \cup\left\{(X \backslash K)^{+}: K \text { compact in } X\right\} \text {; }
$$

so a typical base element for $\tau_{F}$ is of the form $(X \backslash K)^{+} \cap \mathscr{N}^{-}$, where $K \subseteq X$ is compact, and $\mathscr{N}$ is a finite collection of $X$-open sets. We will denote by $c l_{F}(\mathscr{S})$ the $\tau_{F}$-closure of $\mathscr{S} \subseteq C L(X)$. All subspaces of $2^{X}$ will carry the relative Fell topology.

The Fell topology on $2^{X}$ is always compact, and, if $X$ is locally compact, it is also Hausdorff [11]. This in turn implies that $\left(C L(X), \tau_{F}\right)$ is Hausdorff; in fact, $\left(C L(X), \tau_{F}\right)$ is Hausdorff (regular, Tychonoff, respectively) iff $X$ is locally compact [3, Proposition 5.1.2]. Moreover, $\left(C L(X), \tau_{F}\right)$ is normal (paracompact, Lindelöf, respectively) iff $X$ is locally compact, Lindelöf [14, Theorem 1], and $\left(C L(X), \tau_{F}\right)$ is metrizable iff $X$ is locally compact, second countable [3, Theorem 5.1.5]. We will also use that the Fell and the Vietoris topology are admissible, i.e., $X$ embeds as a closed subspace in $\left(C L(X), \tau_{F}\right)$ and $\left(C L(X), \tau_{V}\right)$, respectively. Moreover, if $A \in C L(X)$, then $\left(C L(A), \tau_{F}\right)$ is a closed subspace of $\left(C L(X), \tau_{F}\right)$. The Vietoris and Fell topologies coincide on $C L(X)$ iff $X$ is compact [3].

\section{Embedding into the Fell hyperspace}

Embedding techniques had been successfully used to obtain normality-type results for hyperspaces (see [17] for the Vietoris topology and [7] for the Wijsman topology). In this section, we explore embeddability of various spaces into the Fell hyperspace. 
Recall the definition of the spread and extent, respectively, of a topological space $X$ :

$$
\begin{aligned}
& s(X)=\omega \cdot \sup \{|D|: D \text { is a discrete subspace of } X\}, \\
& e(X)=\omega \cdot \sup \{|D|: D \text { is a closed discrete subspace of } X\} .
\end{aligned}
$$

Proposition 1 If $s(X)>\omega$, then $\left(\omega_{1}+1\right) \times(\omega+1)$ embeds into $\left(2^{X}, \tau_{F}\right)$.

Proof Let $D$ be a discrete subspace of $X$ of size $\omega_{1}, D=\left\{x_{v}: v<\omega_{1}\right\}$. For each $x \in D$, fix an open neighborhood $U(x)$ such that $U(x) \cap D=\{x\}$ and denote by $\preccurlyeq$ the product order on $T_{0}=\left(\omega_{1}+1\right) \times(\omega+1)$ (as usual, $a \prec b$ means that $a \preccurlyeq b$ and $\left.a \neq b\right)$. Let $\Lambda=\left\{\lambda_{\alpha}: 1 \leq \alpha \leq \omega_{1}\right\}$ be the infinite limit ordinals in $\omega_{1}+1$ and put $\lambda_{0}=0$. If $\alpha$ is a successor, denote by $\alpha^{\prime}$ its predecessor.

For convenience, put $\varphi\left(0^{\prime}, \beta\right)=X$ for each $\beta \leq \omega$ and define the function $\varphi: T_{0} \rightarrow 2^{X}$ as follows:

$$
\varphi(\alpha, \beta)= \begin{cases}\varphi\left(\alpha^{\prime}, \beta\right) \backslash \bigcup_{v \in\left[\lambda_{\alpha}, \lambda_{\alpha}+\beta\right]} U\left(x_{\nu}\right) & \text { if }(\alpha, \beta) \in\left(\omega_{1} \backslash \Lambda\right) \times \omega, \\ \bigcap\{\varphi(\bar{\alpha}, \bar{\beta}):(\bar{\alpha}, \bar{\beta}) \prec(\alpha, \beta)\}, & \text { if } \alpha \in \Lambda \text { or } \beta=\omega .\end{cases}
$$

If we take distinct $A, B \in \varphi\left(T_{0}\right)$, then there is $x \in D$ with $U(x)$ missing one of $A, B$ and hitting the other, so $U(x)^{-} \cap \varphi\left(T_{0}\right)$, and $(X \backslash\{x\})^{+} \cap \varphi\left(T_{0}\right)$ are disjoint $\varphi\left(T_{0}\right)$-neighborhoods of $A, B$. Consequently, $\left(\varphi\left(T_{0}\right), \tau_{F}\right)$ is Hausdorff, so to show that $\varphi: T_{0} \rightarrow\left(\varphi\left(T_{0}\right), \tau_{F}\right)$ is a homeomorphism it suffices to show that $\varphi$ is continuous, since $T_{0}$ is compact and $\varphi$ is one-to-one. If $(\alpha, \beta) \in\left(\omega_{1} \backslash \Lambda\right) \times \omega$, then $(\alpha, \beta)$ is isolated in $T_{0}$, so we can assume that one of $\alpha, \beta$ is a limit ordinal. Then, $\varphi(\alpha, \beta)=\bigcap\{\varphi(\bar{\alpha}, \bar{\beta}):(\bar{\alpha}, \bar{\beta}) \prec(\alpha, \beta)\}$, so we just need to consider the $\tau_{F}$-neighborhood $(X \backslash K)^{+} \cap \varphi\left(T_{0}\right)$ of $\varphi(\alpha, \beta)$ for some compact $K \subseteq X$. Then, there exists $(\bar{\alpha}, \bar{\beta}) \prec(\alpha, \beta)$ with $\varphi(\bar{\alpha}, \bar{\beta}) \cap K=\emptyset$, which implies that $\varphi((\bar{\alpha}, \alpha] \times(\bar{\beta}, \beta]) \subset(X \backslash K)^{+} \cap \varphi\left(T_{0}\right)$.

The Tychonoff plank is defined as $T=\left(\omega_{1}+1\right) \times(\omega+1) \backslash\left\{\left(\omega_{1}, \omega\right)\right\}$, where $\omega_{1}+1$ and $\omega+1$ are both endowed with the order topology. It is well known that $T$ is not normal [18].

Corollary 1 1. If $s(X)>\omega$, the Tychonoff plank embeds into $\left(C L(X), \tau_{F}\right)$.

2. If $e(X)>\omega$, the Tychonoff plank embeds into $\left(C L(X), \tau_{F}\right)$ as a closed subspace.

Proof (1) follows from Proposition 1.

(2) If $D$ is an uncountable closed discrete subset of $X$, we can choose $U\left(x_{0}\right)=X \backslash\left(D \backslash\left\{x_{0}\right\}\right)$ in the proof of Proposition 1, so $\varphi\left(\left(\omega_{1}, \omega\right)\right)=\emptyset$. Observe that $A \in C L(X) \backslash \varphi(T)$ iff there exists $(\bar{\alpha}, \bar{\beta}) \prec(\alpha, \beta)$ so that for $i_{1}=\lambda_{\bar{\alpha}}+\bar{\beta}$ and $i_{2}=\lambda_{\alpha}+\beta$ we have $x_{i_{1}} \in A$, $x_{i_{2}} \notin A$. It follows that $U\left(x_{i_{1}}\right)^{-} \cap\left(X \backslash\left\{x_{i_{2}}\right\}\right)^{+}$is a $\tau_{F}$-neighborhood of $A$ disjoint to $\varphi(T)$, so $\varphi(T)$ is a closed subspace of $\left(C L(X), \tau_{F}\right)$.

Proposition 2 Let $\kappa$ be an ordinal and $X$ have a closed discrete set of size $|\kappa|$. Then, $\kappa$ embeds as a closed subspace of $\left(C L(X), \tau_{F}\right)$.

Proof Let $D=\left\{x_{\alpha}: \alpha<\kappa\right\}$ be a closed discrete subspace of $X$, and (without loss of generality) assume $D \neq X$, fix some $x_{-1} \in X \backslash D$. For each $0 \leq \alpha<\kappa$, choose an open set $U\left(x_{\alpha}\right)$ with $\left\{x_{\alpha}\right\}=D \cap U\left(x_{\alpha}\right)$, put $D_{\alpha}=\left\{x_{\beta}: \beta \geq \alpha\right\}$ and define the function $\varphi: \kappa \rightarrow C L(X)$ via $\varphi(\alpha)=D_{\alpha}$. Then, $\varphi$ is clearly injective; moreover,

- $\varphi$ is continuous; indeed, if $\alpha<\kappa$ is a limit ordinal, then $D_{\alpha}=\bigcap_{\beta<\alpha} D_{\beta}$, so it suffices to consider the $\tau_{F}$-neighborhood $(X \backslash K)^{+}$of $\varphi(\alpha)=D_{\alpha}$ for some compact $K \subseteq X$. Then, $D_{\alpha} \cap K=\emptyset$, so $D_{\beta} \cap K=\emptyset$ for some $\beta<\alpha$, i.e., $(\beta, \alpha] \subset \varphi^{-1}\left((X \backslash K)^{+}\right)$. 
- $\varphi$ is open: consider the open set $(\beta, \alpha]$ for some $-1 \leq \beta<\alpha<\kappa$. Then,

$$
D_{\alpha} \in U\left(x_{\alpha}\right)^{-} \cap\left(X \backslash\left\{x_{\beta}\right\}\right)^{+} \cap \varphi(\kappa) \subseteq \varphi((\beta, \alpha]) .
$$

- $\varphi(\kappa)$ is a closed subspace: if $A \in C L(X) \backslash \varphi(\kappa)$, then either $A \backslash D \neq \emptyset$ and then $A \in(X \backslash D)^{-} \subseteq C L(X) \backslash \varphi(\kappa)$ or $A \subset D$ and there exist $0 \leq \beta<\alpha<\kappa$ with $x_{\beta} \in A$, $x_{\alpha} \notin A$, which implies $A \in U\left(x_{\beta}\right)^{-} \cap\left(X \backslash\left\{x_{\alpha}\right\}\right)^{+} \subseteq C L(X) \backslash \varphi(\kappa)$.

Corollary 2 If $s(X)>\omega($ resp. $e(X)>\omega)$, then $\omega_{1}$ and $\omega_{1}+1$ embed in $\left(C L(X), \tau_{F}\right)$ as (closed) subspaces.

Proof See Corollary 1 and Proposition 2.

Corollary 31 . Let $\mathscr{P}$ be a (closed) hereditary topological property that the Tychonoff plank does not have. If $\left(C L(X), \tau_{F}\right)$ has property $\mathscr{P}$, then $s(X)=\omega($ resp. $e(X)=\omega)$.

2. Let $\mathscr{P}$ be a (closed) hereditary topological property that $\omega_{1}$ does not have. If $\left(C L(X), \tau_{F}\right)$ has property $\mathscr{P}$, then $s(X)=\omega($ resp. $e(X)=\omega)$.

Proof Follows from Corollary 1 and Corollary 2.

\section{Normality-related properties of the Fell topology}

Theorem 1 Let $\mathscr{P}$ be a closed hereditary property such that having countable extent with property $\mathscr{P}$ implies Lindelöfness. If $\left(C L(X), \tau_{F}\right)$ has property $\mathscr{P}$, then $X$ is Lindelöf.

Proof The Tychonoff plank (or $\omega_{1}$ ) does not have property $\mathscr{P}$, because it has countable extent and is not Lindelöf. It follows from Corollary 3 that $e(X)=\omega$ and since, by admissibility, $X$ has property $\mathscr{P}$, it is Lindelöf.

Corollary 4 Let $\mathscr{P}$ be a closed hereditary property such that having countable extent with property $\mathscr{P}$ is equivalent to Lindelöfness. Then, the following are equivalent:

1. $\left(C L(X), \tau_{F}\right)$ is $T_{2}$ with property $\mathscr{P}$,

2. $\left(C L(X), \tau_{F}\right)$ is Lindelöf,

3. $X$ is locally compact and Lindelöf.

Proof $(1) \Rightarrow$ (3) follows from Theorem 1 and [3, Proposition 5.1.2], (3) $\Rightarrow(2)$ is known [14, Theorem 1], and $(2) \Rightarrow(1)$ is clear.

The previous results immediately imply characterizations of various properties for the Fell topology, which turn out to be all equivalent to normality of $\left(C L(X), \tau_{F}\right)$ by [14, Theorem 1]. Most of these properties are well known [6]. Recall that $X$ is a $D$-space [2] if for every open neighborhood assignment $N$, one can find a closed discrete $D \subset X$ such that $\{N(x): x \in D\}$ covers $X$; moreover, $X$ is a (weakly) aD-space [2] if for each closed $F \subset X$ (for $F=X$ ) and each open cover $\mathscr{U}$ of $X$, there is a locally finite $A \subset F$ and $\phi: A \rightarrow \mathscr{U}$ with $a \in \phi(a)$ and $F \subset \cup \phi(A)$.

Corollary 5 The following are equivalent:

1. $\left(C L(X), \tau_{F}\right)$ is Lindelöf,

2. $\left(C L(X), \tau_{F}\right)$ is paracompact, 
3. $\left(C L(X), \tau_{F}\right)$ is subparacompact,

4. $\left(C L(X), \tau_{F}\right)$ is metacompact,

5. $\left(C L(X), \tau_{F}\right)$ is submetacompact,

6. $\left(C L(X), \tau_{F}\right)$ is $\sigma$-metacompact,

7. $\left(C L(X), \tau_{F}\right)$ is screenable,

8. $\left(C L(X), \tau_{F}\right)$ is paralindelöf,

9. $\left(C L(X), \tau_{F}\right)$ is $T_{2}$ metalindelöf,

10. $\left(C L(X), \tau_{F}\right)$ is $T_{2}$ submetalindelöf,

11. $\left(C L(X), \tau_{F}\right)$ is a $T_{2} D$-space,

12. $\left(C L(X), \tau_{F}\right)$ is a $T_{2}$ aD-space,

13. $\left(C L(X), \tau_{F}\right)$ is a $T_{2}$ weakly a D-space,

14. $X$ is locally compact and Lindelöf.

Proof Each of the properties (1)-(9) implies (10) (see [6]); (10) implies (13) by [2, Theorem 1.16]. Furthermore, $(11) \Rightarrow(12) \Rightarrow(13)$, and (13) implies (14) by Theorem 1 and [2, Proposition 1.10]. Finally, assuming (14), we get that $\left(C L(X), \tau_{F}\right)$ is $\sigma$-compact by [14, Theorem 1], which yields (1), (11).

Note that all the properties in the previous corollary are not weaker than normality; however, it is known that normality of the Fell topology is equivalent to all of them [14]. We will show that there are properties weaker than normality, which still imply normality for the Fell topology. Recall that a space $X$ is pseudonormal (resp. $\delta$-normal) iff any pair of disjoint closed sets, one of which is countable (resp. a regular $G_{\delta}$ ), can be separated by disjoint open sets.

Theorem 2 The following are equivalent:

1. $\left(C L(X), \tau_{F}\right)$ is a $T_{2}$ countably paracompact space,

2. $\left(C L(X), \tau_{F}\right)$ is a $T_{2} \delta$-normal space,

3. $\left(C L(X), \tau_{F}\right)$ is a $T_{2}$ pseudonormal space,

4. $X$ is locally compact and either countably compact or Lindelöf.

Proof For $(1) \Rightarrow(2)$, see [21, Theorem 3], and for $(2) \Rightarrow(3)$, see [12, Proposition 5.1] using that a Hausdorff Fell hyperspace is Tychonoff as well.

(3) $\Rightarrow(4) X$ is locally compact since $\tau_{F}$ is $T_{2}$. It suffices to prove that if $X$ is not countably compact, then $X$ is Lindelöf; indeed, let $D=\left\{x_{k}: k<\omega\right\}$ be a closed discrete subset of $X$ and define the closed sets $D_{n}=\left\{x_{k}: k \geq n\right\}$ for each $n<\omega$. Then $\mathscr{A}=\left\{D_{n}: n<\omega\right\}$ is a countable $\tau_{F}$-closed set disjoint to the $\tau_{F}$-closed set $\mathscr{B}=\{\{x\}: x \in X\}$. Since $\left(C L(X), \tau_{F}\right)$ is pseudonormal, we can find disjoint $\tau_{F}$-open sets $\mathscr{U}, \mathscr{V}$ so that

$$
\mathscr{A} \subseteq \mathscr{U} \text { and } \mathscr{B} \subseteq \mathscr{V} .
$$

Also, for each $n<\omega$, there exist a finite collection $\mathscr{U}_{n}$ of open sets and a compact $K_{n}$ such that

$$
D_{n} \in\left(X \backslash K_{n}\right)^{+} \cap \mathscr{U}_{n}^{-} \subseteq \mathscr{U} .
$$

We will be done if we show that $X=\bigcup_{n<\omega} K_{n}$ : if there were an $x \in X \backslash \bigcup_{n<\omega} K_{n}$, we could find an open neighborhood $V$ of $x$ and a compact $K$ with

$$
\{x\} \in(X \backslash K)^{+} \cap V^{-} \subseteq \mathscr{V} .
$$

By compactness of $K$, and since $\bigcap_{n<\omega} D_{n}=\emptyset$, this would imply $D_{n} \cap K=\emptyset$ for some $n<\omega$, and hence $D_{n} \cup\{x\} \in \mathscr{U} \cap \mathscr{V}$, a contradiction. 
(4) $\Rightarrow(1)$ If $X$ is countably compact, so is $\left(C L(X), \tau_{F}\right)$ by [13, Proposition 4.1]. If $X$ is locally compact Lindelöf, then $\left(C L(X), \tau_{F}\right)$ is paracompact by [14, Theorem 1].

A space $X$ is weakly normal [1] iff for any pair of disjoint closed sets $A, B \subset X$, there exists a continuous $f: X \rightarrow \mathbb{R}^{\omega}$ so that $f(A), f(B)$ are disjoint. Normality implies weak normality as well as pseudonormality; however, the space constructed in [26, Example 1] is non-normal, weakly normal (since it contains a coarser separable metric space) and pseudonormal. We will show that this distinction is not present for the Fell topology:

Theorem 3 The following are equivalent:

1. $\left(C L(X), \tau_{F}\right)$ is a $T_{2}$ weakly normal, countably paracompact space,

2. $\left(C L(X), \tau_{F}\right)$ is a $T_{2}$ weakly normal, $\delta$-normal space,

3. $\left(C L(X), \tau_{F}\right)$ is a $T_{2}$ weakly normal, pseudonormal space,

4. $\left(C L(X), \tau_{F}\right)$ is normal,

5. $X$ is locally compact and Lindelöf.

Proof Only $(3) \Rightarrow(5)$ needs explanation, the rest follows by Theorem 2 and [14]: if $X$ is countably compact, so is $\left(C L(X), \tau_{F}\right)$ by [13, Proposition 4.1]; moreover, a $T_{2}$ countably compact weakly normal space is normal [1], so $X$ is Lindelöf by [14]. On the other hand, if $X$ is not countably compact, then it must be Lindelöf by Theorem 2 .

The following theorem is the main result of [20]; however, the proof repeatedly uses the incorrect claim that if $A, B$ are disjoint closed subsets of $X$, then $C L(A) \times C L(B)$ embeds as a closed subspace in $C L(A \cup B)$, when the hyperspaces are endowed with the Fell topology (indeed, if $f(C, D)=C \cup D$ is the embedding, then $B \notin f(C L(A) \times C L(B)$ ), but every Fell neighborhood of $B$ intersects $f(C L(A) \times C L(B))$, unless $A$ is compact). We can fix the argument, however, if we work directly inside the Fell hyperspace:

Theorem 4 The following are equivalent:

1. $\left(C L(X), \tau_{F}\right)$ is $T_{2}$ and all of its $F_{\sigma}$-subsets are $\delta$-normal,

2. $\left(C L(X), \tau_{F}\right)$ is normal,

3. $X$ is locally compact and Lindelöf.

Proof $(1) \Rightarrow(3)$ By Theorem 2, we just need to eliminate the possibility that $X$ is countably compact and not Lindelöf; otherwise, $X$ has a countable set $C$ with a limit point $x \notin C$. Let $U \neq X$ be an open neighborhood of $x$ with a compact closure and denote $A=X \backslash U$. We will also assume, without loss of generality, that $\overline{\{x\} \cup C} \subset U$.

Claim If $\mathscr{A} \subseteq C L(A)$ is $\tau_{F}$-closed, then $\mathscr{A}_{D}=\{D \cup B: B \in \mathscr{A}\}$ is $\tau_{F}$-closed for any $D \in U^{+}$.

[Indeed, let $E \in C L(X) \backslash \mathscr{A}_{D}$. Then

- either $E \backslash(D \cup A) \neq \emptyset$, and so $E \in(X \backslash(D \cup A))^{-} \subseteq C L(X) \backslash \mathscr{A}_{D}$,

- or $E \subseteq D \cup A$, then

- either there is $d \in D \backslash E$, and so $E \in(X \backslash\{d\})^{+} \subseteq C L(X) \backslash \mathscr{A}_{D}$,

- or $D \subset E$, and $B=E \cap A \notin \mathscr{A}$. If $(X \backslash K)^{+} \cap \mathscr{N}^{-}$is a $\tau_{F}$-basic neighborhood of $B$ missing $\mathscr{A}$ such that $N \subseteq X \backslash D$ for each $N \in \mathscr{N}$, then so is $(X \backslash K \cap A)^{+} \cap \mathscr{N}^{-}$ which, in turn, is a $\tau_{F}$-neighborhood of $E$ missing $\mathscr{A}_{D}$.] 
Let $\mathscr{A} \subsetneq C L(A)$ be $\tau_{F}$-closed and $\mathscr{W}$ be $C L(A)$-open so that $\mathscr{A} \subseteq \mathscr{W} \subsetneq C L(A)$. Denote $\mathscr{Z}=\mathscr{A}_{\{x\}} \cup \bigcup\left\{C L(A)_{\{c\}}: c \in C\right\}$. Then,

- $\mathscr{B}=\bigcup\left\{(C L(A) \backslash \mathscr{W})_{\{c\}}: c \in C\right\}$ is closed in $\mathscr{Z}:$ if $E \in \mathscr{Z} \backslash \mathscr{B}$, then $E=\{e\} \cup B$ for some $e \in\{x\} \cup C$ and $B \in \mathscr{W}$. Let $(A \backslash K)^{+} \cap \bigcap_{i \leq n}\left(W_{i} \cap A\right)^{-}$be a $C L(A)$-basic neighborhood of $B$ contained in $\mathscr{W}$, where $W_{i} \subseteq X \backslash \overline{\{x\} \cup C}$ for all $i \leq n(n<\omega)$. Then, $(X \backslash K \cap A)^{+} \cap \bigcap_{i \leq n} W_{i}^{-} \cap \mathscr{Z}$ is a $\mathscr{Z}$-neighborhood of $E$ missing $\mathscr{B}$.

- $\mathscr{A}\{x\} \cap \mathscr{B}=\emptyset$ : clear.

- $\mathscr{A}_{\{x\}}$ is a regular $G_{\delta}$ in $\mathscr{Z}$ : for each $c \in C$, let $V_{c}$ be an open neighborhood of $c$ with compact closure such that $\overline{V_{c}}$ misses $A \cup\{x\}$ and denote $\mathscr{O}_{c}=\left(X \backslash \overline{V_{c}}\right)^{+}$. Note that $c l_{F}\left(\mathscr{O}_{c}\right) \subseteq\left(X \backslash V_{c}\right)^{+}$, so

$$
\mathscr{A}\{x\}=\bigcap_{c \in C} \mathscr{Z} \cap \mathscr{O}_{c} \subseteq \bigcap_{c \in C} \mathscr{Z} \cap \operatorname{cl}_{F}\left(\mathscr{O}_{c}\right) \subseteq \bigcap_{c \in C} \mathscr{Z} \cap\left(X \backslash V_{c}\right)^{+}=\mathscr{A}_{\{x\}} .
$$

It follows, by Claim 3, that $\mathscr{Z}$ is an $F_{\sigma}$-subset of $\left(C L(X), \tau_{F}\right)$, and so it is $\delta$-normal; thus, there exist disjoint $\mathscr{Z}$-open sets $\mathscr{U}, \mathscr{V}$ so that $\mathscr{A}_{\{x\}} \subset \mathscr{U}$, and $\mathscr{B} \subset \mathscr{V}$. For each $c \in C$, define the set

$$
\mathscr{U}_{c}=\{B \in C L(A):\{c\} \cup B \in \mathscr{U}\}
$$

Then,

$-c_{C L(A)}\left(\mathscr{U}_{c}\right) \subset \mathscr{W}$; otherwise, if $B \in c_{C L(A)}\left(\mathscr{U}_{c}\right) \backslash \mathscr{W}$, then $\{c\} \cup B \in \mathscr{B} \subset \mathscr{V} \subseteq$ $\mathscr{Z} \backslash \operatorname{cl}_{\mathscr{Z}}(\mathscr{U})$, so there is a $\mathscr{Z}$-neighborhood $(X \backslash K)^{+} \cap \mathscr{N}^{-}$of $\{c\} \cup B$ that misses $\mathscr{U}$. Let $\mathscr{N}_{0}=\left\{N \in \mathscr{N}: B \in N^{-}\right\}$. Then, $(A \backslash K)^{+} \cap \mathscr{N}_{0}^{-}$is a $C L(A)$-neighborhood of $B$ that misses $\mathscr{U}_{c}$, a contradiction.

- $\mathscr{A} \subseteq \bigcup_{c \in C} \mathscr{U}_{c}$ : fix $B \in \mathscr{A}$. Then, there exists a $\tau_{F}$-basic open neighborhood $(X \backslash K)^{+} \cap$ $\mathscr{N}^{-}$of $\{x\} \cup B$ so that $\mathscr{Z} \cap(X \backslash K)^{+} \cap \mathscr{N}^{-} \subseteq \mathscr{U}$. Denote $\mathscr{N}_{1}=\{N \in \mathscr{N}: x \in N\}$. Since $x$ is a cluster point of $C$, we can find a $c \in(C \backslash K) \cap \bigcap \mathscr{N}_{1}$. Then $\{c\} \cup B \in$ $\mathscr{Z} \cap(X \backslash K)^{+} \cap \mathscr{N}^{-} \subseteq \mathscr{U}$, so $B \in \mathscr{U}_{c}$.

It follows by [9, Lemma 1.5.14], that $C L(A)$ is normal; thus, $A=X \backslash U$ is Lindelöf by [14, Theorem 1], and so is $X=(X \backslash U) \cup \bar{U}$, a contradiction.

\section{Metrizability-related properties of the Fell topology}

The point of the following result is to show how various properties of the Vietoris topology provide characterizations of the relevant properties for the Fell topology:

Proposition 3 Let $X$ be a locally compact paracompact space. Let $\mathscr{P}$ be a closed hereditary property such that $\tau_{V}$ has property $\mathscr{P}$ iff $\tau_{V}$ is metrizable. If $\left(C L(X), \tau_{F}\right)$ has property $\mathscr{P}$, then $X$ is metrizable.

Proof Let $K$ be a compact neighborhood of a given $x \in X$. Since the Fell and Vietoris topologies coincide on compacts, and $C L(K)$ is a closed subspace of $\left(C L(X), \tau_{F}\right)$, then $\left(C L(K), \tau_{V}\right)$ has property $\mathscr{P}$, so $K$ is metrizable. It follows that $X$ is locally metrizable so, by the Smirnov metrization theorem $[9,5.4 . \mathrm{A}], X$ is metrizable.

Using the previous result, our last theorem provides characterization of various properties for $\tau_{F}$ that have been separately established for the Vietoris topology [5, 10, 16,25]. 
Theorem 5 The following are equivalent:

1. $\left(C L(X), \tau_{F}\right)$ is $T_{2}$ and a countable union of metrizable subspaces,

2. $\left(C L(X), \tau_{F}\right)$ is $T_{2}$ and symmetrizable,

3. $\left(C L(X), \tau_{F}\right)$ is perfectly normal,

4. $\left(C L(X), \tau_{F}\right)$ is monotonically normal,

5. $\left(C L(X), \tau_{F}\right)$ is hereditarily normal,

6. $\left(C L(X), \tau_{F}\right)$ is metrizable,

7. $X$ is locally compact and 2 nd countable.

Proof (7) $\Leftrightarrow(6)$ follows by [3, Theorem 5.1.5]. Since (6) implies (1)-(5), both (3) and (4) imply (5), we just need to prove that (1), (2), (5), respectively, implies (7):

- (1) implies that $X$ and $\left(C L(X), \tau_{F}\right)$ are $T_{2}$ and locally compact; moreover, $\left(C L(X), \tau_{F}\right)$ is a sequential space by [24, Theorem 1]. This in turn yields that $X$ is hereditarily Lindelöf by [8, Proposition 2.12]. Finally, being a countable union of metrizable subspaces is a property that satisfies Proposition 3 by [16, Corollary 27], so $X$ is metrizable, and (7) follows.

- (2) implies that $\left(C L(X), \tau_{F}\right)$ is $T_{2}$, locally compact and symmetrizable, so it is a Moore space by [6, Theorem 9.13], which is equivalent to (7) by [15, Theorem 7] (an alternative argument could use that $X$ is Lindelöf by [23, Theorem 2], and symmetrizability is a property that satisfies Proposition 3 by [25, Theorem 3]).

- (5) implies that $X$ is locally compact and Lindelöf by [14, Theorem 1], and hereditary normality is another property that satisfies Proposition 3 by [10, Theorem 1], so $X$ is metrizable, and (7) follows.

\section{References}

1. Arhangel'skii, A.V.: Divisibility and cleavability of spaces. In: Recent Developments of General Topology and Its Applications, Mathematical Research 67, pp. 13-26. Akademie, Berlin (1992)

2. Arhangel'skii, A.V.: D-spaces and covering properties. Topol. Appl. 146-147, 437-449 (2005)

3. Beer, G.: Topologies on Closed and Closed Convex Sets. Kluwer, Dordrecht (1993)

4. Beer, G.: On the Fell topology. Set-Valued Anal. 1, 69-80 (1993)

5. Brandsma, H., van Mill, J.: Monotonically normal hyperspaces are metrisable, preprint

6. Burke, D.: Covering properties. In: Kunen, K., Vaughan, J.E. (eds.) Handbook of Set-Theoretic Topology, pp. 347-422. North-Holland, Amsterdam (1984)

7. Cao, J., Junnila, H.J.K., Moors, W.B.: Wijsman hyperspaces: subspaces and embeddings. Topol. Appl. 159, 1620-1624 (2012)

8. Costantini, C., Holá, L., Vitolo, P.: Tightness, character and related properties of hyperspace topologies. Topol. Appl. 142, 245-292 (2004)

9. Engelking, R.: General Topology. Helderman, Berlin (1989)

10. Fedorchuk, V.V.: On Katětov's theorem on the cube. Moscow Univ. Math. Bull. 44, 102-106 (1989)

11. Fell, J.: A Hausdorff topology for the closed subsets of locally compact non-Hausdorff space. Proc. Am. Math. Soc. 13, 472-476 (1962)

12. Good, C., Tree, I.J.: On $\delta$-normality. Topol. Appl. 56, 117-127 (1994)

13. Holá, L., Künzi, H.P.: Properties related to compactness in hyperspaces. Topol. Proc. 23, 191-205 (1998)

14. Holá, L., Levi, S., Pelant, J.: Normality and paracompactness of the Fell topology. J. Proc. Am. Math. Soc. 127, 2193-2197 (1999)

15. Holá, L., Pelant, J., Zsilinszky, L.: Developable hyperspaces are metrizable. Appl. Gen. Topol. 4, 351-360 (2003)

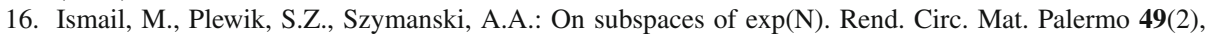
397-414 (2000) 
17. Keesling, J.: On the equivalence of normality and compactness in hyperspaces. Pacific J. Math. 33, 657-667 (1970)

18. Kelley, J.L.: General Topology. Springer, New York (1984)

19. Klein, E., Thompson, A.: Theory of Correspondences. Wiley, New York (1975)

20. Kombarov, A. P.: The $\delta$-Normality of $F_{\sigma}$-Sets in the Fell topology. Math. Notes 92, 720-721 (2012); translated from Mat. Zametki 92, 786-788 (2012)

21. Mack, J.: Countable paracompactness and weak normality properties. Trans. Am. Math. Soc. 148, 265272 (1970)

22. Michael, E.: Topologies on spaces of subsets. Trans. Am. Math. Soc. 71, 152-182 (1951)

23. Nedev, S.: Symmetrizable spaces and final compactness. Dokl. Akad. Nauk SSSR 175, 532-534 (1967)

24. Ostaszewski, A.J.: Compact $\sigma$-metric Hausdorff spaces are sequential. Proc. Am. Math. Soc. 68, 339-343 (1978)

25. Popov, V.V.: Metrizability and the space of closed subsets. Uspekhi Mat. Nauk 35, 209-213 (1980)

26. Reed, G.M.: The intersection topology w.r.t. the real line and the countable ordinals. Trans. Am. Math. Soc. 297, 509-520 (1986)

27. Velichko, N. V.: On spaces of closed subsets. Siberian Math. J. 16, 484-486 (1975); translated from Sibirskii Matem. Z. 16, 627-629 (1975)

28. Zsilinszky, L.: Topological games and hyperspace topologies. Set-Valued Anal. 6, 187-207 (1998) 